\title{
Enseñar y aprender Geografía a partir de distintos len- guajes. Una experiencia de trabajo por proyectos para estudiar el clima de España a partir de los refranes
}

\author{
MARÍA JESÚs MARRÓN GAITE \\ Departamento de Didáctica de las Ciencias Sociales \\ Universidad Complutense de Madrid \\ mjmarron@edu.ucm.es
}

Recibido: 16 de Junio de 2010

Enviado a evaluar: 22 de Julio de 2010

Aceptado: 24 de Febrero de 2011

\section{RESUMEN}

Se presenta una experiencia didáctica sustentada en los fundamentos de la metodología activa y el aprendizaje significativo. A través de ella hemos abordado con estudiantes de Magisterio, en la asignatura "Geografía de España y su Didáctica", el tratamiento del tema "los dominios climáticos de España". Se trata de un diseño didáctico enmarcado en la técnica del "trabajo por proyectos de investigación", tomando como elemento motivador y núcleo de interés el análisis de algunos refranes alusivos al tema, seleccionados del amplio y rico refranero español. A partir de cada uno de ellos los estudiantes formulan las hipótesis del trabajo de investigación correspondientes a cada bloque de contenidos.

Con este trabajo queremos aportar algunas ideas acerca de cómo puede llevarse a cabo la enseñanza-aprendizaje de la Geografía de forma práctica, motivadora y eficaz a partir de un tipo de lenguaje que a los estudiantes les resulta atractivo y motivador: los refranes.

Palabras clave: Geografía, enseñanza activa, trabajo por proyectos, investigación, refranes

\section{Geography teaching and learning from different languages. Work experience of project to study the climate of Spain from the proverbs}

\begin{abstract}
We present a learning experience underpinned by the fundamentals of the methodology active and meaningful learning. Through it we have dealt with student teachers in the course "Geography of Spain and its Didactics", treatment of the theme "climate domains of Spain." This is an instructional design technique framed "research project work," taking as a motivator and focus of interest the analysis of some proverbs alluding to the theme, selected the broad and rich Spanish proverbs. Since each student formulate research hypotheses for each content block.
\end{abstract}


In this paper we provide some ideas about how you can take out the teaching and learning of geography in a practical, motivating and effective from a kind of language that students find it attractive and motivating: proverbs.

Key words: Geography, active learning, project work, research, proverbs.

Enseignement de la Geographie et l'apprentissage des langues differentes. Expérience de travail du projet pour etudier le climat de l'Espagne aux proverbes

\section{RÉSUMÉ}

Nous présentons une expérience d'apprentissage repose sur les principes fondamentaux de la méthodologie d'apprentissage active et significative. Par elle, nous avons traité avec les enseignants des élèves dans le cours "Géographie de l'Espagne et sa didactique", domaines du traitement du thème "climat de l'Espagne." Il s'agit d'une technique de conception pédagogique encadrée "de travail du projet de recherche", en prenant comme source de motivation et de centre d'intérêt l'analyse de certains proverbes faisant allusion au thème, sélectionnez les proverbes large et riche en espagnol. Étant donné que chaque élève de formuler des hypothèses de recherche pour chaque bloc de contenu.

Dans cet article nous donner quelques idées sur la façon dont vous pouvez retirer de l'enseignement et l'apprentissage de la géographie dans une pratique, motivant et efficace à partir d'un type de langage que les élèves trouvent proverbes attirante et motivante.

Mots-clés: Géographie, l'apprentissage actif, des projets, la recherche, des proverbes

\section{INTRODUCCIÓN}

La experiencia didáctica que presentamos en el presente trabajo surgió como consecuencia de una doble convicción que siempre hemos tenido en relación con la enseñanza de la Geografía. Por una parte, de la necesidad que existe de abordar la enseñanza-aprendizaje de los contenidos geográficos desde la perspectiva de la metodología activa y participativa, potenciando el aprendizaje autónomo de los estudiantes. Y por otra, de la necesidad que existe, si queremos alcanzar resultados satisfactorios, de estimular en los alumnos la motivación intrínseca hacia el conocimiento y el gusto por aprender a aprender.

A través de ella hemos abordado con alumnos de Magisterio, en la asignatura "Geografía de España y su Didáctica", el tratamiento del tema "los dominios climáticos de España". Se trata de un diseño didáctico enmarcado en el método de "trabajo por proyectos de investigación", tomando como elemento motivador y núcleo de interés el análisis de algunos refranes alusivos al tema, seleccionados del amplio y rico refranero español. En torno a este núcleo se vertebran los contenidos que se van a trabajar.

Los refranes constituyen una forma de sabiduría popular, basada en la observación y la experiencia individual y colectiva de múltiples fenómenos de diversa tipo- 
logía: naturales, sociales, económicos, comportamentales, etc. Son el resultado de observaciones repetidas por múltiples personas a lo largo de muchos siglos, de ahí, el alto grado de veracidad que entrañan. La mayoría de ellos se cumplen en un elevado porcentaje de casos, lo cual no debe extrañarnos, ya que el refrán que no se cumple con una alta regularidad acaba desapareciendo. Infinidad de refranes atestiguan con sus sentencias lo que decimos. Sirvan como referencia los siguientes:

- No hay refrán que no sea verdadero.

- Cien refranes, cien verdades.

- No hay refrán que no diga una verdad; y si una no, es porque dice dos.

- Refrán no verdadero, poco duradero.

No debe extrañarnos, por tanto, que sus aseveraciones formen, con frecuencia, parte de ese saber práctico que rige la actividad de determinados colectivos humanos. Especialmente dado a los refranes ha sido siempre el medio rural, en el que la sabiduría popular ha plasmado mediante sus sentencias, elaboradas a través de siglos, distintas reflexiones acerca de aquellos aspectos de la vida que más les preocupaban. Una de las principales inquietudes de las gentes del campo ha sido siempre el tiempo atmosférico y los ritmos e intensidad con los que precipitaciones, temperaturas y vientos se dan sobre un determinado lugar, pues ello condiciona de forma decisiva el éxito o el fracaso de las cosechas. De hecho, la mayor parte de los refranes sobre el tiempo tienen su origen en el medio rural y con frecuencia vinculan meteorología y agricultura.

Por todo lo expuesto, consideramos que utilizar como centro de interés, para el tratamiento del tema propuesto, una selección de refranes de los muchos que existen en el refranero español sobre el tiempo atmosférico, constituye un recurso didáctico interesante y útil para trabajar los contenidos del tema, al tiempo que permite enfocarlo desde una perspectiva multidisciplinar, integrando contenidos geográficos, lingüísticos, históricos, antropológicos, etc.

Con esta propuesta queremos aportar algunas ideas acerca de cómo puede llevarse a cabo la enseñanza-aprendizaje de la Geografía de forma práctica, amena y eficaz a partir de un tipo de lenguaje que a los estudiantes les resulta atractivo y motivador: los refranes.

\section{LOS REFRANES COMO RECURSO DIDÁCTICO EN LA ENSEÑANZA ACTIVA DE LA GEOGRAFÍA}

En las últimas décadas el valor de la enseñanza activa, basada en el constructivismo y el aprendizaje significativo, ha sido propugnado por las corrientes psicológicas y pedagógicas más innovadoras, que buscan en ella un revulsivo frente a la enseñanza memorística y libresca. Estas corrientes recogen los postulados de la Escuela Nueva, defensora del aprendizaje experiencial, basado en el contacto con el medio más cercano, para a partir de su conocimiento abordar contenidos a distintas escalas. Los presupuestos pedagógicos de la Escuela Nueva instan al profesorado a fomentar la curiosidad de los estudiantes despertando su atención por los fenómenos 
de la Naturaleza, al tiempo que proponen la indagación como metodología de aprendizaje y la resolución de problemas como estrategia didáctica.

Partiendo de estos postulados entendemos que los refranes pueden ser empleados didácticamente como centro de interés para el aprendizaje de infinidad de contenidos curriculares pertenecientes a las distintas disciplinas académicas. En el caso de la Geografía resultan especialmente útiles para el tratamiento de un elevado número de contenidos tanto de Geografía Física como Humana. La ciencia geográfica, situada en la encrucijada de lo físico y lo social, estudia cómo es el mundo en esa doble vertiente. Nos enseña a conocer y valorar el resultado de la interacción entre el hombre y el medio que habita y de las sociedades con la Naturaleza; interacción que a lo largo del tiempo ha ido conformando la enorme diversidad de culturas que existen o han existido sobre el planeta, las cuales, a su vez imprimen su impronta particular sobre el medio físico que ocupan, dando lugar a distintas formas paisajísticas.

Los refranes que hacen alusión a aspectos del medio físico o a las interacciones que se dan entre éste y la acción antrópica son numerosísimos. Especialmente abundantes son los referidos al tema objeto de estudio en este trabajo: "los dominios climáticos de España", los cuales son el resultado de una observación continuada sobre los distintos fenómenos atmosféricos que se suceden en un determinado lugar y de las consecuencias que de ellos se derivan.

Las posibilidades de aprovechamiento didáctico que ofrecen los refranes para el tratamiento del tema que nos ocupa son múltiples. Con el fin de no resultar prolijos, señalamos a continuación las más significativas:

- La interpretación que ofrecen de la realidad a través de sus sentencias, permite formular hipótesis de partida para trabajar múltiples contenidos curriculares propios del tema que nos ocupa. Por ejemplo, el refrán "en abril aguas mil", se interpreta como que el mes de abril es uno de los meses del año que registra mayores índices de precipitación, y que ésta se produce en forma de lluvia. Esta sentencia, transformada en hipótesis puede constituir la base de la labor de investigación, conducente a verificar, recurriendo a las fuentes pertinentes, el grado de veracidad de lo que en él se afirma.

- Permiten abordar el estudio de los contenidos de forma globalizada. Por ejemplo, el refrán "agua por San Juan, quita vino, aceite y pan", se interpreta como que no es normal que llueva cuando el mes de junio está muy avanzado (San Juan es el 24 de mayo). Pero, también nos indica que estas lluvias son perjudiciales para la vid, el olivar y el trigal. Por ello, además de permitirnos tratar los aspectos meteorológicos pertinentes, nos permite analizar una amplia gama de contenidos referidos a la agricultura mediterránea. El hecho de que se aluda expresamente a estos tres cultivos, nos lleva a preguntarnos acerca de la importanica que tienen en el agro mediterráneo. Al mismo tiempo, nos llevará a estudiar el relevante papel que esta trilogía agraria ha jugado en el mudo mediterráneo a lo largo de la Historia y en la actualidad, desde el punto de vista económico, social, religioso y cultural. Todo ello favorece el aprendizaje globalizado e integrador de los distintos tipos de contenidos, los cuales adquieren significación al vincularse entre sí. 
- Constituyen un elemento altamente motivador. Esta virtualidad reside, por una parte, en la singularidad de su estructura sintáctica y el ritmo de su cadencia, que les hace simpáticos y atractivos, fáciles de retener y de recordar. Por otra, en el alto porcentaje de veracidad que sus sentencias revisten, reflejo de una sabiduría popular muy intuitiva y fácil de comprender.

- Acercan a los estudiantes al conocimiento de múltiples contenidos geográficos de forma amena y sugerente. Los refranes presentan el objeto de su reflexión de forma ingeniosa y atractiva.

- Favorecen la capacidad de observación. Al ser los refranes sentencias sobre hechos observados durante largo tiempo, inducen a la comprobación de los fenómenos a los que aluden, para lo cual se hace necesaria la observación de los mismos. Sólo después de la fase de observación vendrá la formulación de hipótesis y la verificación de las mismas.

- Potencian el desarrollo del pensamiento analítico y la capacidad de síntesis. Sus asertos son interpretaciones de la realidad sobre la que se pronuncian dotados de una fuerte capacidad de penetración y análisis. Así mismo, el resultado de ese análisis se expresa de una forma extremadamente breve y concisa, mediante una frase ingeniosa a la vez que contundente.

\section{EL TRABAJO POR PROYECTOS EN LA ENSEÑANZA ACTIVA DE LA GEOGRAFÍA}

\subsection{EL TRABAJO POR PROYECTOS}

Los fundamentos didácticos del modelo de enseñanza por proyectos de investigación se sustentan en la filosofía de la Escuela Nueva, que concede un valor capital al aprendizaje basado en la experiencia personal del individuo y a la indagación e investigación personalizada conforme al método científico. De ahí la eficacia que su empleo reviste para la enseñanza activa de las distintas disciplinas académicas, y especialmente de la Geografía, debido al carácter de ciencia de síntesis que ésta tiene y a las múltiples variables que se impone analizar al estudiar los hechos y fenómenos geográficos, tanto físicos como derivados de la interacción entre el medio físico y la acción antrópica.

Especialmente significativo para la implantación y desarrollo de esta estrategia fue el llamado Plan Dalton, que supuso una gran aportación didáctica al ofrecer una solución práctica a los ritmos individuales de aprendizaje y cultivar la iniciativa personal del alumno, que gozaba de libertad para elegir el momento en que debía trabajar y el proceso que iba a seguir en su tarea de aprendizaje. Unido a esta práctica de la libertad individual estaba el fomento de la responsabilidad de cada alumno hacia su propio trabajo, que tenía lugar en los llamados laboratorios, en cada uno de los cuales se abordaba el tratamiento de las distintas disciplinas del currículo de forma interrelacionada.

Este modelo se complementa a partir del Sistema Winetka, en el que se armoniza el trabajo individual con el trabajo en equipo. La programación se establece a partir de un listado de conocimientos y técnicas que los alumnos deben ir adquiriendo con- 
forme a sus necesidades para la vida. En él se articulan una serie de etapas y pasos, al tiempo que se contemplan las características psicológicas de cada educando.

Dewey, filósofo y pedagogo estadounidense, transciende los trabajos anteriores y postula la necesidad de unir la escuela con la vida, concediendo un valor fundamental a la experiencia, concebida como transacción entre el sujeto y su entorno. Para él, la educación es la posibilidad de acrecentar experiencias, y la escuela es una sociedad en pequeño en cuyas aulas tiene lugar una vida comunitaria que permite a cada sujeto aumentar su experiencia social. A partir de esta idea concibe un modelo de trabajo denominado método de proyectos, entendiendo como proyectos la actividad espontánea y ordenada que realiza un grupo de alumnos que previamente ha elegido un tema de estudio. Sus discípulos y seguidores desarrollaron y experimentaron esta metodología de forma sistemática y sus estudios se difundieron por toda la comunidad científica. Hoy día es un método que goza de un enorme prestigio asociado al espíritu de la renovación e innovación pedagógica.

Kilpatrick, discípulo de Dewey, definió el método por proyectos como "un acto problemático llevado a su completa realización como un acto natural" $(1951,168)$ queriendo significar con ello que un proceso complejo como es la investigación a través del método científico, se convierte en algo natural y fácil de realizar si se parte de la idea esencial que caracteriza a este método: el objeto de estudio es elegido vinculándolo a los intereses del alumnado y relacionándolo con su realidad vivencial. Con posterioridad, Beaumont y Williams (1983, 1), citando a Gool (1973) han matizado más el concepto, entendiendo como proyecto "una unidad significativa y práctica de actividad, dotada de valor educativo y enfocada hacia una o más metas definidas de comprensión; implica investigación y resolución de problemas planificada y culminada hasta su resolución por el alumno y el profesor de un modo natural como en la vida real". De esta definición podemos extraer varios rasgos que distinguen al método por proyectos de formas más librescas y convencionales de enseñanza-aprendizaje. Podemos destacar:

- Opta por la reflexión frente al aprendizaje memorístico.

- Concede prioridad a los problemas frente a los principios.

- Contextualización realista del aprendizaje. Vinculado a la vida real.

- Carácter eminentemente activo, desarrollando habilidades y destrezas.

- Potencia el aprendizaje personalizado, adaptado a los ritmos de cada alumno.

\subsection{APLICACIONES DIDÁCTICAS DEL TRABAJO POR PROYECTOS EN LA ENSENAANZA- APRENDIZAJE DE LA GEOGRAFÍA}

El empleo didáctico del trabajo por proyectos en Geografía implica una concepción innovadora acerca de cómo ha de enfocarse la acción educativa y de cómo entender los procesos de enseñanza-aprendizaje de esta disciplina. Supone apostar por una metodología activa, global e integradora, que potencie el aprendizaje multidisciplinar, favorezca el aprendizaje completo (el saber, el saber hacer y el saber ser) y capacite a los estudiantes para comprender la multicausalidad de los procesos espaciales y la valoración ponderada de las variables que los configuran. En esta metodología el 
protagonista del aprendizaje será siempre el propio alumno, quien, partiendo de los conceptos previos que integran su acerbo cultural, irá progresando en la construcción de su propio saber de forma personalizada, conducente a alcanzar aprendizajes significativos. Este modo de hacer se fundamenta en los principios de la enseñanza acti$v a$, en la cual el educando no puede considerarse a sí mismo ni ser considerado como un mero receptor de contenidos elaborados, sino que, por el contrario, ha de participar activamente en el proceso de aprendizaje, tanto a través de actuaciones individuales como mediante la participación en equipos de trabajo y en la realización de proyectos compartidos. Potencia en el alumno el interés por entender el mundo y las cosas, le ayuda a transferir el conocimiento de los contenidos, que trabaja académicamente, a la vida real y favorece el desarrollo de la motivación intrínseca.

Desde esta concepción metodológica las estrategias a emplear serán fundamentalmente la indagación y la investigación orientadas por el profesor. Ahora bien, existen numerosos contenidos de carácter conceptual y procedimental que el alumno no es capaz de elaborar por sí mismo a partir de los preconceptos que posee, por lo que se impone combinar el aprendizaje por descubrimiento y autoconstrucción dirigidos con el empleo de técnicas transmisivas o expositivas, enmarcadas, eso sí, dentro de un enfoque investigativo. La indagación y la investigación deberán constituir, como ha señalado GARDNER (2002) estrategias de aprendizaje básicas en todos los niveles de enseñanza. A través de ellas el educando se inicia en el método científico de estudio y análisis de la realidad socioespacial, practicando la observación (directa e indirecta), formulando hipótesis, manejando fuentes, analizando resultados y elaborando conclusiones de forma personalizada, en función de su capacidad de conceptualización y desde sus propios intereses y motivaciones.

Las ventajas didácticas que esta estrategia metodológica ofrece para la enseñanzaaprendizaje de la Geografía son múltiples. Señalamos de forma sintética las siguientes:

1) Favorece el aprendizaje activo y significativo de los contenidos geográficos a través del trabajo personalizado.

2) Desarrolla la capacidad para identificar y definir los aspectos más relevantes de cualquier hecho o proceso con componente espacial.

3) Propicia la explicación multicausal de los fenómenos espaciales y la valoración de las variables que los configuran.

4) Favorece el aprendizaje de los contenidos geográficos en los tres ámbitos del conocimiento: conceptual, procedimental y actitudinal.

5) Potencia el desarrollo de las habilidades implicadas en la labor de investigación y permite conocer los problemas que en ella pueden plantearse.

6) Genera la satisfacción propia del trabajo creativo, ya que los resultados obtenidos son la consecuencia de una labor de investigación personal, que permite aportar nueva información sobre el tema objeto de estudio.

7) Fomenta el sentido de responsabilidad y compromiso personal ante la tarea que se ha asumido realizar.

Al mismo tiempo el trabajo por proyectos de investigación permite a los alumnos "adquirir las habilidades adecuadas para interpretar múltiples aspectos de la rea- 
lidad próxima y remota y los fines y usos de esas habilidades se mantienen vivos en la conciencia del educando a lo largo de toda su vida ... mediante ellos las disciplinas no se trabajan de una forma aislada, que proporciona poca motivación, sino más bien, como parte de una implicación continuada para abordar temas que resuenan en todo el currículo escolar ... y desarrollar las inteligencias múltiples que toda persona posee" Gardner $(2003,128)$.

\section{UNA EXPERIENCIA DE TRABAJO POR PROYECTOS: ESTUDIO DE LOS DOMINIOS CLIMÁTICOS DE ESPAÑA A PARTIR DE LOS REFRANES}

En este apartado exponemos una experiencia de investigación empírica basada en el trabajo por proyectos de investigación, empleando como elemento motivador y núcleo de interés una selección de refranes alusivos al tema objeto de estudio.

Se trata de un trabajo llevado a la práctica con estudiantes de Magisterio en la asignatura "Geografía España y su Didáctica" para estudiar el tema de "los dominios climáticos de España". Con la realización del mismo se persigue propiciar el aprendizaje de los contenidos geográficos propios del tema de forma activa, desarrollando estrategias de enseñanza-aprendizaje basadas en la indagación y la investigación empírica. Se trata de potenciar en los estudiantes el interés por el conocimiento científico de la realidad a partir de las sentencias de los refranes, que al transformarlas en hipótesis de trabajo les permitan investigar de acuerdo con el Método Científico. Partiendo de las hipótesis formuladas se estudian los diferentes factores que configuran al clima de cada uno de los dominios climáticos de España. Así mismo, a partir de un conjunto de refranes alusivos a las repercusiones que los fenómenos meteorológicos tienen en la actividad agraria, se abordará de forma complementaria la incidencia que el clima tiene en la agricultura. Los contenidos del tema se trabajan de forma globalizada y multidisciplinar, atendiendo a su tratamiento desde los tres ámbitos del conocimiento: conceptual, procedimental y actitudinal.

El proyecto está estructurado en seis módulos, cada uno de los cuales corresponde a uno de los dominios climáticos de nuestro país. Para su realización se divide el grupo-clase en equipos de trabajo, encargándose cada uno del estudio de un dominio climático. Realizado el tratamiento de cada módulo, se procede a la puesta en común y al análisis comparativo de los resultados obtenidos por cada equipo de trabajo. Todos los bloques tienen la misma estructura y se trabajan con la misma metodología, acorde con el Método Científico de investigación. Debido a la extensión que supondría la presentación del diseño completo del trabajo, exponemos a modo de ejemplo uno de los módulos, el correspondiente a "la España seca".

\subsection{ESTUDIO DEL DOMINIO CLIMÁTICO “LA ESPAÑA SECA” A PARTIR DE LOS REFRANES.}

\subsubsection{OBJETIVOS}

- Conocer la delimitación espacial del dominio climático correspondiente a la España seca. 
- Ejercitarse en la observación de los fenómenos meteorológicos que caracterizan a los tipos de tiempo que determinan el clima de la zona.

- Identificar y definir los aspectos más relevantes del clima y el tiempo atmosférico de este dominio climático.

- Aprender a formular hipótesis con rigor científico.

- Desarrollar destrezas para el análisis de las fuentes que se van a emplear: lingüísticas, documentales, cartográficas y orales.

- Aprender a organizar, interpretar y utilizar científicamente la información extraída de dichas fuentes.

- Profundizar en el estudio del tema mediante una metodología activa y participativa, que permita alcanzar aprendizajes significativos.

- Propiciar la explicación multicausal, analizando el conjunto de variables que contribuyen a la configuración de este dominio climático.

- Dominar el conocimiento del tema desde los tres ámbitos del conocimiento: conceptual, procedimental y actitudinal.

- Valorar críticamente la información que sobre el tiempo atmosférico y el clima ofrecen los refranes seleccionados y ver en que modo se cumplen o no en este dominio climático.

- Analizar el valor didáctico que ciertos refranes tienen como elemento motivador para estudiar este tema.

- Despertar el interés por el trabajo creativo.

- Fomentar el sentido de responsabilidad y compromiso personal ante la tarea que se ha asumido realizar y generar la satisfacción por el trabajo bien hecho.

- Despertar el interés por el aprendizaje basado en el método por proyectos de investigación.

\subsubsection{CONTENIDOS}

1. Aspectos generales del ámbito de estudio

1.1. Localización y delimitación espacial

1.2. Extensión y altitud media.

1.3. Configuración física

2. El clima

2.1. Análisis de las temperaturas

2.1.1. Distribución anual de las temperaturas.

2.1.1. Temperaturas medias

2.1.2. Temperaturas absolutas: máximas y mínimas.

2.2. Análisis de las precipitaciones.

2.2.1. Precipitación anual

2.2.2. Tipología de las precipitaciones

2.2.2.1. Por su origen

2.2.2.2. Por su naturaleza 
2.3. Régimen de vientos

2.3.1. Vientos dominantes

2.3.2. Vientos dominantes en los días de lluvia

2.3.3. Vientos dominantes en los días secos

3. Clima y agricultura

3.1. Cultivos dominantes

3.1.1. El policultivo de secano

3.1.1.1. Cultivo cerealista de "año y vez"

3.1.1.2. Cultivo vitícola.

3.1.1.3. El olivar

3.1.1.4. Los frutales de secano

3.1.2. Cultivos de regadío

3.1.2.1. Cultivos industriales

3.1.2.2. Cultivos forrajeros

3.1.2.3. Cultivos hortícolas

3.2. Los cultivos de invernadero

3.3. El clima y el calendario agrícola

3.2.1. Adaptación de las labores agrícolas al ritmo estacional

3.2.2. Labores agrícolas propias de cada estación

El tratamiento de estos contenidos se lleva a cabo tomando como núcleo de interés un conjunto de refranes, que se han seleccionado en base a las características climáticas de la "España seca". Han sido extraídos del Refranero General Ideológico Español, compilado por Matínez Kleiser, en la edición facsímil de la editorial Hernando de 1988. Se han elegido refranes alusivos a los tres parámetros que definen el clima: temperaturas, precipitaciones y régimen de vientos.

El estudio del clima se complementa con el análisis de la influencia que éste ejerce sobre las actividades humanas, concretamente sobre la agricultura. Para ello, se han seleccionado algunos refranes que aluden de forma expresa a esta influencia.

* Refranes propuestos para el estudio del clima:

A) Relativos a las temperaturas:

- En enero se hiela el agua en el caldero

- En febrero el loco ningún día se parece al otro

- En marzo la veleta ni dos horas está quieta.

- En hora buena vengáis mayo, el mejor mes del año

- Marzo ventoso y abril lluvioso, preparan a mayo florido y hermoso.

- Hasta el cuarenta de mayo no te quites el sayo.

- No hay verano hasta San Juan (24 de junio), ni invierno hasta Navidad.

- En julio, beber y sudar, y el fresco en balde buscar

- En las madrugadas de agosto ha frío el viejo y el mozo

- En octubre caída de hojas y lumbre

- Por Todos los Santos (1 de noviembre), nieve en los altos 
- En diciembre no hay valiente que no tiemble

- En diciembre, días de amargura: apenas amanece, ya es noche oscura

- Gran calma y gran calura, agua segura.

B) Relativos a las precipitaciones:

- Año de nieves, año de bienes

- Lluvia menuda y caladera, es la que quiere la tierra.

- Con nieve en enero, no hay año fulero

- Las aguas marceras son muy hierberas

- En abril, aguas mil.

- Agua de mayo no cala el sayo; y si alguna vez lo caló, pronto se enjugó

- Julio normal, seca el manantial

- Septiembre o lleva los puentes o seca las fuentes

- Octubre las mejores frutas pudre

* Refranes que relacionan el tiempo atmosférico y la agricultura:

- Eche agua Dios, que es oro para nos (para el labrador)

- Con agua no hay tierra mala.

- Agua y sol, dos grandes amigos del labrador

- El granizo empobrece y no encarece

- Si en Pascua mucho llueve, póngase el labrador alegre

- Semana Santa mojada, cuartilla de trigo colmada

- Llueva por abril y mayo y no llueva en todo el año

- Agua de mayo, pan para todo el año.

- Agua en junio, infortunio

- Agua por San Juan, quita aceite, vino y pan

- Si en julio llueve, renace la hierba y el trigo se pierde

- Cuando el invierno es lluvioso, el verano es abundoso

\subsubsection{HIPÓTESIS}

La interpretación de cada uno de los refranes permite formular una o varias hipótesis, a partir de las cuales se inicia el trabajo de investigación.

El objetivo esencial del trabajo, no es tanto comprobar en que medida las hipótesis de partida se cumplen, como propiciar que los alumnos lleguen al conocimiento del tema a partir de ellas, mediante la acción investigadora. Exponemos, a continuación, algunos ejemplos.

- En marzo la veleta ni dos horas está quieta.

Indica que marzo se caracteriza por los días ventosos. Los alumnos formularán hipótesis acerca del por qué de esta característica de marzo, que les llevará a estudiar los mecanismos de la circulación atmosférica, la formación de frentes, su representación cartográfica, etc.

- En diciembre no hay valiente que no tiemble

- En diciembre, días de amargura: apenas amanece, ya es noche oscura 
El primero indica que las temperaturas en este mes del año son muy bajas. El segundo, hace referencia a que los días son muy cortos. A partir de ellos se puede trabajar: análisis de datos relativos a valores de temperaturas medias, de máximas y mínimas absolutas, su evolución a lo largo del año, amplitud térmica anual, repercusiones sobre la vegetación (natural y cultivos), realización e interpretación de gráficas térmicas, etc.

- En abril, aguas mil.

- Julio normal, seca el manantial

El primero se interpreta como que abril es uno de los meses del año con más precipitación. El segundo indica que julio se caracteriza por la sequía hidrológica. A partir de ellos se trabaja: el análisis de datos pluviométricos, su distribución a lo largo del año, análisis de series anuales, tipos de precipitación, estudio conjunto de precipitación y temperaturas, repercusiones en el medio físico y en las actividades humanas, etc.

- Eche agua Dios, que es oro para nos (para el labrador)

- Con agua no hay tierra mala.

Estos dos refranes indican el valor que tiene el agua para el agricultor en la denominada España seca, donde este elemento es tan escaso debido a la precariedad de las precipitaciones. A partir de ellos, además de trabajarse los contenidos sobre precipitaciones, se trabaja: la influencia que el clima tiene en la agricultura, los cultivos dominantes en la zona, la morfología agraria, los paisajes agrarios de la España seca, etc.

- El granizo empobrece y no encarece.

Se interpreta que el granizo es muy perjudicial para el campo porque daña los cultivos, esto supone pérdidas para el agricultor. Dado que el granizo está asociado siempre a las tormentas, a partir de este refrán se trabajan: las precipitaciones de carácter convectivo, las causas y las consecuencias tan perjudiciales de las mismas (en el medio físico y en la actividad agraria), etc.

\subsubsection{FUENTES}

Dadas las características de la investigación y del tema objeto de estudio, las fuentes empleadas son de tres tipos:

\section{- Lingüísticas:}

Diccionario de la Real Academia Española de la Lengua.

Refranero General Ideológico Español, compilado por MATÍNEZ KLEISER

\section{- Cartográficas:}

Mapa de los dominios climáticos de España. Escala 1/200.000

Mapa de cultivos y usos del suelo agrario. Escala 1/200.000

Mapa Topográfico Nacional. Hojas del área de estudio. Escala 1/50.000

\section{- Referidas a las variables climáticas}

Instituto Nacional de Meteorología: datos sobre temperaturas, precipitaciones y régimen de vientos de varias Estaciones Meteorológicas ubicadas en el dominio climático objeto de estudio. Incluimos a modo de ilustración los datos de algunas de ellas (Tablas 1 a 8 ). 


\section{BIBLIOGRAFÍA}

AYLLÓN, T. y GUTIERREZ ROA, J. ( 1989): Introducción a la observación meteorológica. México: Limusa.

ALBENTOSA SÁNCHEZ, L. (1988): El clima y las aguas. Madrid: Síntesis. Colección Geografía de España.

BARRY, R.G. y CHORLEY, R.J. (1980): Atmósfera, tiempo y clima. Omega: Barcelona.

BEAUMONT, J.R. y WILLIANS, S.W. (1983): Proyect work in the geography curriculum. An advanced level primer. Londres: Croom Helm.

CONONI, M. et al. (1987): Metodología activa. Reflexiones-Experiencias. Madrid: Acción Educativa.

DUNI, S. y WILSON, F. (2001): Cómo predecir el tiempo. Guía del meteorólogo aficionado. Madrid: CEAC.

ESCOURROU, G. (1988): Climatologie pratique. París: Masson

ESTÉBANEZ ÁlVAREZ, J. (1988): "Los espacios rurales" En R. PUYOL, J. ESTÉBANEZ y R. MÉNDEZ: Geografia Humana. Madrid: Cátedra, pp. 239-356.

FAUCHHER, D. (1985): Geografia agraria. Tipos de cultivos. Barcelona: Omega.

FERNÁNDEZ DE ARRÓYABE HERNÁEZ, P. (1999): "Evaluación del valor científico de los refranes como fuentes indirectas de información climática". Lurralde, 22, pp. 323-337.

GARDNER, H. (1987): Estructuras de la mente. La teoría de las múltiples inteligencias. Méjico: Fondo de Cultura Económica.

GARDNER, H. (2003): Inteligencias múltiples. La teoría en la práctica. Barcelona: Paidós.

GILBANK, G. (1974): Introduction à la Geographie Générale de l'Agriculture. Paris : Masson.

GIL, A. (1982): De la agricultura tradicional a la tecnológica. Madrid: Cincel.

GREGOR, H. (1987): Geografía de la agricultura. Barcelona: Vicens Vives.

KILPATRICK, W.H. (1951): Filosofia de la educación. Nueva York: Macmillan.

LACOSTE, Y. y GHIRARDI, R. (1983): Geografia General. Física y Humana. Barcelona. Oikos-Tau.

LEBEAU, R. (1983): Grandes modelos de estructuras agrarias en el mundo. Barcelona: Vicens Vives.

MEDINA, M. (1980): Iniciación a la meteorología. Madrid: Paraninfo.

MARRÓN GAITE, M ${ }^{\mathrm{a}}$ J. (Ed.)(2001): La educación geográfica de los ciudadanos en el cambio de milenio. Madrid: Grupo de Didáctica de la Geografía de la Asociación de Geógrafos Españoles y Universidad Complutense.

MARRON GAITE, M ${ }^{\mathrm{a}}$ J. (2003): "La educación geográfica en el siglo XXI". En MARRÓN GAITE; MORALEDA NIETO y RODRÍGUEZ DE GRACIA (Eds.): $L a$ enseñanza de la Geografía ante las nuevas demandas sociales. Toledo: Grupo de Didáctica de la Geografía de la Asociación de Geógrafos Españoles y Universidad de Castilla-La Mancha (EU de Magisterio de Toledo), pp. 259-275.

MARRÓN GAITE, M ${ }^{\mathrm{a}}$ J. y GARCÍA FERNÁNDEZ, G. (Eds.) (2004): Agricultura, medio ambiente y sociedad. Madrid: Ministerio de Agricultura. Serie Estudios.

MARTÍNEZ KLEISER, L. (1988): Refranero General Ideológico Español. Edicción Facsimil. Madrid: Hernando. 
MORENO JIMÉNEZ, A. (1995): "Enseñar investigando: el modelo de proyectos de investigación". En A. MORENO JIMÉNEZ y M.J. MARRÓN GAITE: Enseñar Geografía. De la teoría a la práctica. Madrid: Síntesis, pp. 121-138.

MORGAN, W.B. y MUNTON, R.J.C. (1985): Geografia agrícola. Barcelona: Omega.

PEÑA, O. (1982): Diccionario de Climatología. Valparaíso: Universidad Católica de Valparaíso.

PLATA SUAREZ, J. (2005): "Errores geográficos que se transmiten en las instituciones escolares”. Didáctica Geográfica. 7, pp. 447-462.

SÁEZ LORITE, M. y FERRER RODRÍGUEZ, A. (1988): Los paisajes rurales. Madrid: Síntesis. Colección Geografía de España.

SANCHO, J. (1982): La agricultura de cara al futuro. Madrid: Cincel.

SPEDDING, C.R.W. (1993): Ecología de los sistemas agrícolas. Barcelona: Blume.

SPITZ, A. (1983): El tiempo. Introducción a la meteorología. Barcelona: Bruguera.

STRALHER, A. (1977): Geografía Física. Barcelona: Omega.

TERÁN, M., SOLÉ SABARÍS, L. et al. (1978): Geografía General de España. Barcelona: Ariel.

TOHARIA, M. (1973): El libro del tiempo. Meteorología, refranes y contaminación. Madrid: Ed. Noventa y Nueve.

TOHARIA, M. (1985): Meteorología popular. Madrid: El Observatorio.

TOHARIA, M. (1981): Tiempo y clima. Barcelona: Salvat. Temas Clave.

VV.AA. (1980): Los paisajes rurales de España. Valladolid: Asociación de Geógrafos Españoles y Universidad de Valladolid.

VICUÑA, G. (1999): Manual de meteorología popular. Edicción Facsimil. Palma de Mallorca: Librería Naútica.

VILÁ VALENTÍ, J. (1989): La Península Ibérica. Barcelona: Ariel.

VILÁ VALENTÍ, J. (1990): Geografía de España. Barcelona: Danae. 
Tabla 1. Distribución de las temperaturas anuales en Carrión de los Condes (Palencia), 1990

\begin{tabular}{|c|c|c|c|c|c|c|c|c|c|c|c|}
\hline \multirow{3}{*}{ Meses } & \multirow{3}{*}{$\begin{array}{l}\text { Temp. }{ }^{\circ} \mathrm{C} \\
\text { Máxima } \\
\text { Absoluta }\end{array}$} & \multirow{3}{*}{ Fechas } & \multirow{3}{*}{$\begin{array}{l}\text { Temp. }{ }^{\circ} \mathrm{C} \\
\text { Mínima } \\
\text { absoluta }\end{array}$} & \multirow{3}{*}{ Fechas } & \multicolumn{3}{|c|}{ Temperaturas medias en ${ }^{\circ} \mathrm{C}$} & \multicolumn{4}{|c|}{ Número de días de temperaturas } \\
\hline & & & & & \multirow{2}{*}{ Máxima } & \multirow{2}{*}{ Mínima } & \multirow{2}{*}{ Mensual } & \multicolumn{2}{|c|}{ Mínimas absolutas } & \multicolumn{2}{|c|}{ Máximas absolut. } \\
\hline & & & & & & & & $</=0^{\circ} \mathrm{C}$ & $>/=20^{\circ} \mathrm{C}$ & $>/=25^{\circ} \mathrm{C}$ & $>/=30^{\circ} \mathrm{C}$ \\
\hline Enero & 16,0 & 27 & $-7,0$ & Varias & 7,3 & $-0,1$ & 3,6 & 18 & 0 & 0 & 0 \\
\hline Febrero & 15,0 & Varias & $-4,5$ & 12 & 11,3 & 1,6 & 6,4 & 10 & 0 & 0 & 0 \\
\hline Marzo & 23,0 & 31 & $-4,0$ & 14 & 12,5 & 1,4 & 6,9 & 14 & 0 & 0 & 0 \\
\hline Abril & 23,5 & 1 & $-3,0$ & 8 & 14,6 & 3,7 & 9,2 & 6 & 0 & 0 & 0 \\
\hline Mayo & 25,0 & 11 & 4,0 & Varias & 17,9 & 6,6 & 12,2 & 0 & 0 & 1 & 0 \\
\hline Junio & 32,0 & 4 & 6,5 & Varias & 23,5 & 8,6 & 16,0 & 0 & 0 & 10 & 3 \\
\hline Julio & 37,0 & 24 & 4,0 & 17 & 29,6 & 9,7 & 19,7 & 0 & 0 & 26 & 17 \\
\hline Agosto & 38,0 & 22 & 8,0 & 1 & 31,4 & 12,5 & 21,9 & 0 & 0 & 30 & 24 \\
\hline Sept. & 34,0 & 2 & 5,0 & 24 & 27,5 & 9,6 & 18,6 & 0 & 0 & 24 & 11 \\
\hline Octubre & 30,0 & 1 & $-3,0$ & 20 & 18,3 & 6,0 & 12,1 & 3 & 0 & 6 & 1 \\
\hline Nov. & 25,0 & 1 & $-6,5$ & 30 & 11,4 & 1,1 & 6,2 & 16 & 0 & 1 & 0 \\
\hline Dic. & 15,5 & 23 & $-11,5$ & 9 & 7,9 & $-4,4$ & 1,8 & 29 & 0 & 0 & 0 \\
\hline Año & 38,0 & $\begin{array}{c}22 \mathrm{de} \\
\text { Agosto }\end{array}$ & $-11,5$ & $\begin{array}{l}9 \mathrm{de} \\
\text { Dic. }\end{array}$ & 17,8 & 4,7 & 11,2 & 96 días & 0 días & 98 días & 56 días \\
\hline
\end{tabular}

Fuente: Instituto Nacional de Meteorología

Tabla 2. Precipitaciones y régimen de vientos en Carrión de los Condes (Palencia), 1990

\begin{tabular}{|c|c|c|c|c|c|c|c|c|}
\hline \multirow{3}{*}{ Meses } & \multicolumn{3}{|c|}{ Precipitación } & \multirow{3}{*}{$\begin{array}{l}\text { Viento dominante en los } \\
\text { días con precipitación }\end{array}$} & \multicolumn{4}{|c|}{ Número de días de } \\
\hline & \multirow{2}{*}{ Total (mm.) } & \multicolumn{2}{|c|}{ Máxima en 24 horas } & & \multirow{2}{*}{ Lluvia } & \multirow{2}{*}{ Nieve } & \multirow{2}{*}{ Granizo } & \multirow{2}{*}{ Tormenta } \\
\hline & & $\mathrm{mm}$. & Fechas & & & & & \\
\hline Enero & 10,8 & 3,8 & 19 & NW & 7 & 4 & 0 & 0 \\
\hline Febrero & 61,8 & 34,5 & 25 & $\mathrm{~W}$ & 8 & 0 & 0 & 0 \\
\hline Marzo & 61,7 & 27,8 & 27 & $\mathrm{~S}$ & 9 & 1 & 1 & 0 \\
\hline Abril & 64,5 & 25,5 & 14 & $\mathrm{~S}$ & 8 & 0 & 0 & 1 \\
\hline Mayo & 47,7 & 17,4 & 12 & $\mathrm{~S}$ & 10 & 0 & 0 & 3 \\
\hline Junio & 18,9 & 8,7 & 11 & $\mathrm{~W}$ & 6 & 0 & 2 & 1 \\
\hline Julio & 3,4 & 2,8 & 15 & $\mathrm{~W}$ & 2 & 0 & 1 & 2 \\
\hline Agosto & 35,5 & 14,0 & 23 & $\mathrm{E}$ & 5 & 0 & 0 & 3 \\
\hline Sept. & 5,4 & 4,0 & 19 & $\mathrm{~W}$ & 2 & 0 & 0 & 0 \\
\hline Octubre & 28,8 & 19,5 & 15 & SW & 6 & 0 & 0 & 0 \\
\hline Nov. & 32,4 & 14,3 & 11 & SW & 7 & 3 & 0 & 0 \\
\hline Dic. & 0,0 & 0,0 & - & - & - & 0 & 0 & 0 \\
\hline Año & 370,9 & 34,5 & 21 de Abril & - & 72 & 8 & 4 & 10 \\
\hline
\end{tabular}

Fuente: Instituto Nacional de Meteorología

Tabla 3. Distribución de las temperaturas anuales de Sardón de Duero (Valladolid), 2001

\begin{tabular}{|c|c|c|c|c|c|c|c|c|c|c|c|}
\hline \multirow{3}{*}{ Meses } & \multirow{3}{*}{$\begin{array}{l}\text { Temp. }{ }^{\circ} \mathrm{C} \\
\text { Máxima } \\
\text { Absoluta }\end{array}$} & \multirow{3}{*}{ Fechas } & \multirow{3}{*}{$\begin{array}{l}\text { Temp. }{ }^{\circ} \mathrm{C} \\
\text { Mínima } \\
\text { absoluta }\end{array}$} & \multirow{3}{*}{ Fechas } & \multicolumn{3}{|c|}{ Temperaturas medias en ${ }^{\circ} \mathrm{C}$} & \multicolumn{4}{|c|}{ Número de días de temperaturas } \\
\hline & & & & & \multirow{2}{*}{ Máxima } & \multirow{2}{*}{ Mínima } & \multirow{2}{*}{ Mensual } & \multicolumn{2}{|c|}{ Mínimas absolutas } & \multicolumn{2}{|c|}{ Máximas absolut. } \\
\hline & & & & & & & & $</=0{ }^{\circ} \mathrm{C}$ & $>/=20^{\circ} \mathrm{C}$ & $>/=25^{\circ} \mathrm{C}$ & $>/=30^{\circ} \mathrm{C}$ \\
\hline Enero & 16,0 & 27 & $-8,0$ & 15 & 8,0 & $-0,7$ & 3,6 & 23 & 0 & 0 & 0 \\
\hline Febrero & 16,5 & 4 & $-5,0$ & 11 & 11,8 & 0,1 & 5,9 & 16 & 0 & 0 & 0 \\
\hline Marzo & 23,0 & 31 & $-6,0$ & 16 & 12,5 & 0,8 & 6,7 & 14 & 0 & 0 & 0 \\
\hline Abril & 23,5 & 1 & $-2,5$ & Varias & 14,6 & 1,5 & 8,1 & 16 & 0 & 0 & 0 \\
\hline Mayo & 23,0 & 11 & 0,0 & 22 & 16,8 & 4,4 & 10,6 & 1 & 0 & 0 & 0 \\
\hline Junio & 30,5 & 4 & 3,5 & 28 & 22,8 & 7,5 & 15,1 & 0 & 0 & 8 & 2 \\
\hline Julio & 36,5 & 22 & 2,5 & 10 & 28,8 & 9,0 & 18,7 & 0 & 0 & 24 & 16 \\
\hline Agosto & 37,0 & 23 & 7,0 & 8 & 30,3 & 11,2 & 20,7 & 0 & 0 & 28 & 20 \\
\hline Sept. & 33,5 & 7 & 6,0 & 10 & 28,0 & 9,6 & 18,8 & 0 & 0 & 24 & 12 \\
\hline Octubre & 28,5 & 1 & $-2,5$ & 20 & 19,0 & 4,5 & 11,7 & 4 & 0 & 5 & 0 \\
\hline Nov. & 24,5 & 1 & $-6,0$ & 30 & 12,0 & 0,0 & 6,0 & 18 & 0 & 0 & 0 \\
\hline Dic. & 14,0 & 14 & 10,5 & 30 & 8,7 & $-5,0$ & 1,9 & 27 & 0 & 0 & 0 \\
\hline Año & 37,0 & 22 de Agosto & $-10,0$ & 30 de Dic. & 17,7 & 3,6 & 10,6 & 119 días & 0 & 89 días & 50 días \\
\hline
\end{tabular}

Fuente: Instituto Nacional de Meteorología

2011, vol. 31, núm. 1 107-123 
Tabla 4. Precipitaciones y régimen de vientos en Sardón de Duero (Valladolid), 2001.

\begin{tabular}{|c|c|c|c|c|c|c|c|c|}
\hline \multirow{3}{*}{ Meses } & \multicolumn{3}{|c|}{ Precipitación } & \multirow{3}{*}{$\begin{array}{l}\text { Viento dominante en los } \\
\text { días con precipitación }\end{array}$} & \multicolumn{4}{|c|}{ Número de días de } \\
\hline & \multirow{2}{*}{ Total (mm.) } & \multicolumn{2}{|c|}{ Máxima en 24 horas } & & \multirow{2}{*}{ Lluvia } & \multirow{2}{*}{ Nieve } & \multirow{2}{*}{ Granizo } & \multirow{2}{*}{ Tormenta } \\
\hline & & $\mathrm{mm}$. & Fechas & & & & & \\
\hline Enero & 16,7 & 4,0 & 24 & SW & 9 & 3 & 0 & 0 \\
\hline Febrero & 24,0 & 10,0 & 15 & $\mathrm{NE}$ & 9 & 0 & 0 & 0 \\
\hline Marzo & 46,1 & 19,0 & 21 & $\mathrm{NE}$ & 10 & 3 & 2 & 0 \\
\hline Abril & 73,1 & 22,9 & 15 & SW & 7 & 1 & 0 & 0 \\
\hline Mayo & 49,2 & 19,0 & 12 & SW & 16 & 0 & 0 & 2 \\
\hline Junio & 42,2 & 32,7 & 11 & SW & 6 & 0 & 1 & 1 \\
\hline Julio & 3,1 & 2,0 & 8 & SW & 0 & 0 & 1 & 3 \\
\hline Agosto & 8,9 & 3,0 & 10 & SW & 1 & 0 & 1 & 2 \\
\hline Sept. & 21,5 & 14,5 & 19 & SW & 5 & 0 & 0 & 0 \\
\hline Octubre & 24,7 & 11,8 & 23 & SW & 8 & 0 & 0 & 0 \\
\hline Nov. & 35,7 & 10,4 & 11 & SW & 9 & 2 & 1 & 0 \\
\hline Dic. & 8,8 & 5,0 & 14 & SW & 4 & 1 & 0 & 0 \\
\hline Año & 354,0 & 32,7 & 11 de Junio & SW & 84 & 10 & 6 & 8 \\
\hline
\end{tabular}

Fuente: Instituto Nacional de Meteorología.

Tabla 5. Distribución de las temperaturas anuales en Alcázar de San Juan (Ciudad Real), 1985

\begin{tabular}{|c|c|c|c|c|c|c|c|c|c|c|c|}
\hline \multirow{3}{*}{ Meses } & \multirow{3}{*}{$\begin{array}{l}\text { Temp. }{ }^{\circ} \mathrm{C} \\
\text { Máxima } \\
\text { Absoluta }\end{array}$} & \multirow{3}{*}{ Fechas } & \multirow{3}{*}{$\begin{array}{l}\text { Temp. }{ }^{\circ} \mathrm{C} \\
\text { Mínima } \\
\text { absoluta }\end{array}$} & \multirow{3}{*}{ Fechas } & \multicolumn{3}{|c|}{ Temperaturas medias en ${ }^{\circ} \mathrm{C}$} & \multicolumn{4}{|c|}{ Número de días de temperaturas } \\
\hline & & & & & \multirow{2}{*}{ Máxima } & \multirow{2}{*}{ Mínima } & \multirow{2}{*}{ Mensual } & \multicolumn{2}{|c|}{ Mínimas absolutas } & \multicolumn{2}{|c|}{ Máximas absolut. } \\
\hline & & & & & & & & $</=0^{\circ} \mathrm{C}$ & $>/=20^{\circ} \mathrm{C}$ & $>/=25^{\circ} \mathrm{C}$ & $>/=30^{\circ} \mathrm{C}$ \\
\hline Enero & 16,4 & 30 & $-4,8$ & 4 & 8,5 & $-0,2$ & 4,1 & 12 & 0 & 0 & 0 \\
\hline Febrero & 20,9 & 18 & $-2,8$ & Varias & 11,7 & 1,6 & 6,6 & 9 & 0 & 0 & 0 \\
\hline Marzo & 23,6 & 29 & $-2,1$ & 9 & 12,9 & 2,7 & 7,8 & 7 & 0 & 0 & 0 \\
\hline Abril & 26,6 & 25 & 0,9 & 3 & 17,3 & 5,1 & 11,2 & 1 & 0 & 0 & 0 \\
\hline Mayo & 33,1 & 31 & 5,8 & 6 & 23,3 & 9,4 & 16,4 & 0 & 0 & 1 & 0 \\
\hline Junio & 36,9 & Varias & 7,2 & Varias & 28,3 & 12,8 & 20,6 & 0 & 0 & 2 & 9 \\
\hline Julio & 40,1 & Varias & 11,0 & 10 & 32,8 & 15,5 & 24,2 & 0 & 0 & 6 & 21 \\
\hline Agosto & 39,2 & Varias & 10,6 & 2 & 32,4 & 15,4 & 23,9 & 0 & 0 & 4 & 20 \\
\hline Sept. & 35,4 & 9 & 6,9 & 4 & 27,5 & 12,3 & 19,9 & 0 & 0 & 0 & 15 \\
\hline Octubre & 27,1 & 12 & 1,8 & 6 & 19,6 & 7,7 & 13,6 & 1 & 0 & 0 & 3 \\
\hline Nov. & 20,8 & 2 & $-1,7$ & 12 & 21,1 & 3,5 & 7,8 & 9 & 0 & 0 & 0 \\
\hline Dic. & 15,3 & 1 & $-3,5$ & 9 & 9,3 & 0,5 & 4,9 & 13 & 0 & 0 & 0 \\
\hline Año & 40,1 & 18 de Julio & $-4,8$ & 3 de Enero & 19,8 & 4,7 & 24,2 & 52 días & 0 días & 98 días & 56 días \\
\hline
\end{tabular}

Fuente: Instituto Nacional de Meteorología

Tabla 6. Precipitaciones y régimen de vientos en Alcázar de San Juan (Ciudad Real), 1985

\begin{tabular}{|c|c|c|c|c|c|c|c|c|}
\hline \multirow{3}{*}{ Meses } & \multicolumn{3}{|c|}{ Precipitación } & \multirow{3}{*}{ Viento dominante en los días con precipitación } & \multicolumn{4}{|c|}{ Número de días de } \\
\hline & \multirow{2}{*}{ Total (mm.) } & \multicolumn{2}{|c|}{ Máxima en 24 horas } & & \multirow{2}{*}{ Lluvia } & \multirow{2}{*}{ Nieve } & \multirow{2}{*}{ Granizo } & \multirow{2}{*}{ Tormenta } \\
\hline & & $\mathrm{mm}$. & Fechas & & & & & \\
\hline Enero & 37,7 & 11,1 & 8 & SW & 5 & 0 & 0 & 0 \\
\hline Febrero & 46,0 & 12,0 & 12 & SW & 7 & 0 & 0 & 0 \\
\hline Marzo & 36,0 & 17,0 & 19 & SW & 4 & 0 & 3 & 0 \\
\hline Abril & 55,1 & 21,3 & 12 & SW & 16 & 0 & 0 & 0 \\
\hline Mayo & 41,2 & 17,1 & 10 & $\mathrm{~S}$ & 6 & 0 & 0 & 2 \\
\hline Junio & 30,3 & 27,2 & 22 & S & 3 & 0 & 2 & 2 \\
\hline Julio & 4,0 & 2,3 & 10 & SW & 1 & 0 & 1 & 3 \\
\hline Agosto & 12,1 & 3,0 & 26 & SW & 2 & 0 & 1 & 4 \\
\hline Sept. & 22,3 & 13,5 & 18 & SW & 5 & 0 & 0 & 0 \\
\hline Octubre & 37,2 & 11,9 & 27 & SW & 9 & 0 & 0 & 0 \\
\hline Nov. & 42,0 & 11,2 & 12 & SW & 10 & 0 & 3 & 0 \\
\hline Dic. & 50,1 & 20,6 & 23 & SW & 6 & 1 & 0 & 0 \\
\hline Año & 424,0 & 32,7 & 11 de Junio & SW & 74 & 1 & 10 & 11 \\
\hline
\end{tabular}

Fuente: Instituto Nacional de Meteorología 
Tabla 7. Distribución de las temperaturas anuales en Torrijos (Toledo), 1987

\begin{tabular}{|c|c|c|c|c|c|c|c|c|c|c|c|}
\hline \multirow{3}{*}{ Meses } & \multirow{3}{*}{$\begin{array}{l}\text { Temp. }{ }^{\circ} \mathrm{C} \\
\text { Máxima } \\
\text { Absoluta }\end{array}$} & \multirow{3}{*}{ Fechas } & \multirow{3}{*}{$\begin{array}{l}\text { Temp. }{ }^{\circ} \mathrm{C} \\
\text { Mínima } \\
\text { absoluta }\end{array}$} & \multirow{3}{*}{ Fechas } & \multicolumn{3}{|c|}{ Temperaturas medias en ${ }^{\circ} \mathrm{C}$} & \multicolumn{4}{|c|}{ Número de días de temperaturas } \\
\hline & & & & & \multirow{2}{*}{ Máxima } & \multirow{2}{*}{ Mínima } & \multirow{2}{*}{ Mensual } & \multicolumn{2}{|c|}{ Mínimas absolutas } & \multicolumn{2}{|c|}{ Máximas absolut. } \\
\hline & & & & & & & & $</=0^{\circ} \mathrm{C}$ & $>/=20^{\circ} \mathrm{C}$ & $>/=25^{\circ} \mathrm{C}$ & $>1=30^{\circ} \mathrm{C}$ \\
\hline Enero & 16,7 & 27 & $-6,9$ & 29 & 9,7 & $-1,3$ & 4,2 & 13 & 0 & 0 & 0 \\
\hline Febrero & 17,7 & 20 & $-5,8$ & 3 & 11,7 & $-0,6$ & 5,6 & 8 & 0 & 0 & 0 \\
\hline Marzo & 22,0 & 19 & $-2,8$ & 5 & 16,0 & 2,3 & 9,1 & 9 & 0 & 0 & 0 \\
\hline Abril & 26,1 & 24 & 0,0 & 2 & 20,2 & 4,8 & 12,5 & 2 & 0 & 0 & 0 \\
\hline Mayo & 30,6 & 29 & 1,9 & 3 & 23,2 & 8,1 & 15,7 & 0 & 2 & 1 & 0 \\
\hline Junio & 35,8 & 27 & 6,4 & 5 & 29,5 & 13,2 & 21,3 & 0 & 4 & 12 & 9 \\
\hline Julio & 39,1 & 14 & 11,3 & 1 & 33,8 & 16,5 & 25,1 & 0 & 4 & 26 & 21 \\
\hline Agosto & 37,9 & Varias & 11,2 & 27 & 33,2 & 15,9 & 24,5 & 0 & 5 & 25 & 20 \\
\hline Sept. & 34,7 & Varias & 6,7 & 12 & 28,4 & 12,2 & 20,3 & 0 & 0 & 26 & 15 \\
\hline Octubre & 26,3 & 10 & 0,6 & 22 & 20,2 & 6,7 & 13,4 & 0 & 0 & 9 & 3 \\
\hline Nov. & 19,0 & 3 & 1,7 & 26 & 12,0 & 2,2 & 8,1 & 0 & 0 & 4 & 0 \\
\hline Dic. & 14,8 & 2 & 4,7 & 28 & 9,9 & $-0,6$ & 4,7 & 0 & 0 & 0 & 0 \\
\hline Año & 39,1 & 14 de Julio & $-6,9$ & 29 de Enero & 20,8 & 6,6 & 13,7 & & & & \\
\hline
\end{tabular}

Fuente: Instituto Nacional de Meteorología

Tabla 8. Precipitaciones y régimen de vientos en Torrijos (Toledo), 1987

\begin{tabular}{|c|c|c|c|c|c|c|c|c|}
\hline \multirow{3}{*}{ Meses } & \multicolumn{3}{|c|}{ Precipitación } & \multirow{3}{*}{ Viento dominante en los días con precipitación } & \multicolumn{4}{|c|}{ Número de días de } \\
\hline & \multirow{2}{*}{ Total (mm.) } & \multicolumn{2}{|c|}{ Máxima en 24 horas } & & \multirow{2}{*}{ Lluvia } & \multirow{2}{*}{ Nieve } & \multirow{2}{*}{ Granizo } & \multirow{2}{*}{ Tormenta } \\
\hline & & $\mathrm{mm}$. & Fechas & & & & & \\
\hline Enero & 39,0 & 10,1 & 8 & NW & 6 & 0 & 0 & 0 \\
\hline Febrero & 42,2 & 12,9 & 12 & NW & 6 & 0 & 0 & 0 \\
\hline Marzo & 40,0 & 15,0 & 19 & NW & 7 & 0 & 1 & 0 \\
\hline Abril & 47,0 & 21,3 & 12 & NW & 10 & 0 & 0 & 0 \\
\hline Mayo & 41,3 & 16,2 & 10 & $\mathrm{~S}$ & 5 & 0 & 2 & 3 \\
\hline Junio & 35,2 & 15,2 & 22 & $\mathrm{~S}$ & 5 & 0 & 3 & 2 \\
\hline Julio & 6,0 & 2,5 & 10 & SW & 1 & 0 & 1 & 4 \\
\hline Agosto & 8,4 & 3,1 & 26 & $\mathrm{E}$ & 1 & 0 & 1 & 3 \\
\hline Sept. & 37,2 & 12,5 & 18 & $\mathrm{E}$ & 5 & 0 & 0 & 0 \\
\hline Octubre & 54,0 & 19,8 & 27 & $\mathrm{E}$ & 6 & 0 & 0 & 0 \\
\hline Nov. & 50,7 & 17,4 & 12 & NW & 11 & 0 & 1 & 0 \\
\hline Dic. & 48,2 & 13,3 & 23 & NW & 7 & 0 & 0 & 0 \\
\hline Año & 449,2 & 21,3 & 12 de Abril & NW & 70 & 0 & 9 & 12 \\
\hline
\end{tabular}

Fuente: Instituto Nacional de Meteorología 